\title{
What's Old is New Again
}

\section{Editorial about "Total Thyroidectomy is Associated with Increased Risk of Complications for Low- and High-Volume Surgeons," by Hauch et al., doi:10.1245/s10434-014-3846-8}

\author{
Julie Ann Sosa, MD, MA, FACS
}

Section of Endocrine Surgery, Endocrine Neoplasia Diseases Group, Department of Surgery, Duke Cancer Institute and Duke Clinical Research Institute, Duke University, Durham, NC

In "Total thyroidectomy is associated with increased risk of complications for low-and high-volume surgeons," Hauch et al. demonstrate in the current issue of Annals of Surgical Oncology just that; total thyroidectomy implies there is more surgery than thyroid lobectomy with attendant increased risk of postoperative morbidity, and highvolume surgeons on average perform thyroid surgery with improved outcomes compared to low-volume colleagues.

The association between increased provider volume and improved patient outcomes is by no means a novel concept, and it has been shown to be a robust one in multiple surgical specialties, for many different procedures, and across many different datasets. Sometimes, the association is between patient outcomes and surgeon volume, and other times it is with hospital volume. In endocrine surgery, individual surgeon volume was initially demonstrated to be associated independently with complication rates and length of stay for thyroidectomy by Sosa et al. ${ }^{1}$ using hospital discharge data from the Maryland Health Services Cost Review Commission. A very small proportion of all thyroid surgeons were high-volume, and they performed a disproportionately large proportion of thyroidectomies, but still the overwhelming majority of patients with surgical thyroid disease underwent surgery by less experienced surgeons, raising the question of compromised access leading to variation in the quality of care. The relation was shown in disease and procedure subgroups; indeed, patients with thyroid cancer who

(C) Society of Surgical Oncology 2014

First Received: 26 May 2014;

Published Online: 19 June 2014

J. A. Sosa, MD, MA, FACS

e-mail: Julie.sosa@duke.edu underwent total thyroidectomy and substernal thyroidectomy demonstrated the most exquisite association between their surgeons' volume and outcomes.

Since then, the association has been demonstrated to be a robust one, and similar findings have been replicated in pediatric and geriatric thyroid patients, as well as in inpatient and ambulatory settings on a large scale. ${ }^{2-5}$ Therefore, the data from Hauch et al. are confirmatory but by no means novel. Whereas their conclusions support those of many other investigators, specific findings have somewhat limited interpretability and generalizability. Charges rather than costs are reported, and adjustment is not made for inflation; these are relative deficiencies that can be overcome by employing hospital-specific cost to charge ratios and then adjusting costs for inflation using an inflation rate available from the Bureau of Labor Statistics. This is unfortunate, because it impairs our ability to interpret these data in the crowded landscape of similar published studies. Another unstated limitation of the analysis is that generalizability is compromised by the fact that the Nationwide Inpatient Sample is restricted to inpatient procedures; recent studies have demonstrated that the majority of thyroidectomies in the United States are performed now in the ambulatory setting. ${ }^{6}$ This is the likely explanation for the high absolute rate of complications reported, because there is likely selection bias related to patients undergoing inpatient procedures, which are likely for more severe or complex disease.

However, this study is an important one for its implications in the renewed debate about the appropriate management for low-and medium-risk differentiated thyroid cancers $<4 \mathrm{~cm}$ in size, and whether thyroid lobectomy or total thyroidectomy should be performed, and in the case 
of papillary thyroid microcarcinomas (PTMCs), whether surgery needs to be performed at all. In 2009, the American Thyroid Association issued guidelines for the management of thyroid nodules and differentiated thyroid cancer which recommended that tumors $>1 \mathrm{~cm}$ undergo total or neartotal thyroidectomy, and PTMCs at least undergo ipsilateral thyroid lobectomy if they were deemed to be low risk. ${ }^{7}$ The rationale for the former recommendation was that total thyroidectomy was shown to be associated with improvement in survival compared to thyroid lobectomy, and total thyroidectomy could be performed safely, especially by high-volume surgeons. ${ }^{8}$ Since then, however, a larger body of evidence using large, population-level datasets has mounted demonstrating equivalent survival between the two surgical approaches. ${ }^{9-11}$ Debate continues about recurrence rates, but here, too, evidence is emerging calling into some question previously observed differences. ${ }^{11,12} \mathrm{In}$ addition, longer term follow-up is available showing that active surveillance is an acceptable alternative to surgery for patients with low-risk tumors $<1 \mathrm{~cm}$ in size. ${ }^{13}$ Finally, routine postoperative use of radioactive iodine ablation (RAI) is evolving into a more selective approach to ablation in patients with low-and intermediate-risk cancers, taking away this rationale for total thyroidectomy.

The study by Hauch et al. demonstrates in a compelling fashion that total and near-total thyroidectomy, indeed, are associated with a significantly higher rate of short-term postoperative complications compared with thyroid lobectomy, including hypocalcemia and neck hematoma, and this difference in morbidity is observed in the hands of both low-and high-volume surgeons. Overall morbidity is understated, in that the analysis does not take into consideration the quality of life implications involved with life-long thyroid hormone supplementation and/or replacement following surgery, or postdischarge morbidity, such as readmission. At a population level, on average, total thyroidectomy appears to be less safe short-term than thyroid lobectomy, regardless of the case volume of the operating surgeon.

In the end, counseling patients about the relative risks and benefits of thyroid lobectomy versus total thyroidectomy will necessitate essential data such as that provided by Hauch et al.; the decision for many patients with lowand intermediate-risk tumors $<4 \mathrm{~cm}$ in size will rest on fastidious preoperative risk stratification and patient preference.

What will the new guidelines from the American Thyroid Association recommend? We should know later in 2014. They will need to be informed by data such as these.

DISCLOSURES There are no relevant disclosures.

\section{REFERENCES}

1. Sosa JA, Bowman HM, Tielsch JM, et al. The importance of surgeon experience for clinical and economic outcomes from thyroidectomy. Ann Surg. 1998;228(3):320-330.

2. Tuggle CT, Roman SA, Wang TS, et al. Pediatric endocrine surgery: who is operating on our children? Surgery. 2008;144(6): 869-877.

3. Sosa JA, Mehta P, Wang TS, et al. A population based study of outcomes from thyroidectomy in aging Americans: At what cost? J Am Coll Surg. 2008;206(3):1097-1105.

4. Kandil E, Noureldine SI, Abbas A, et al. The impact of surgical volume on patient outcomes following thyroid surgery. Surgery. 2013;154(6):1346-1352.

5. Tuggle CT, Roman S, Udelsman R, et al. Same day thyroidectomy: a review of practice patterns and outcomes for 1168 procedures in New York State. Ann Surg Oncol. 2011;18(4):1035-1040.

6. Sosa JA, Hanna JW, Robinson KA, et al. Increases in thyroid nodule fine-needle aspirations, operations, and diagnoses of thyroid cancer in the United States. Surgery. 2013;154(6):1420-1426.

7. Cooper DS, Doherty GM, Haugen BR, et al. Revised American Thyroid Association management guidelines for patients with thyroid nodules and differentiated thyroid cancer. Thyroid. 2009;19(11):1167-1214.

8. Bilimoria KY, Bentrem DJ, Ko CY, et al. Extent of surgery affects survival for papillary thyroid cancer. Ann Surg. 2007; 246(3):375-581.

9. Mendelsohn AH, Elashoff DA, Abemayor E, et al. Surgery for papillary thyroid carcinoma: Is lobectomy enough? Arch Otolaryngol Head Neck Surg. 2010;136(11):1055-1061.

10. Haigh PI, Urbach DR, Rotstein LE. Extent of thyroidectomy is not a major determinant of survival in low-or high-risk of papillary thyroid cancer. Ann Surg Oncol. 2005;12(1):81-89.

11. Nixon IJ, Ganley I, Patel SG, et al. Thyroid lobectomy for treatment of well differentiated intrathyroid malignancy. Surgery. 2012;151(4):571-579.

12. Matsuzu K, Sugino K, Masudo K, et al. Thyroid lobectomy for papillary thyroid cancer: long term follow up study of 1088 cases. World J Surg. 2014;38(1):68-79.

13. Ito Y, Miyauchi A, Kihara M, et al. Patient age is significantly related to the progression of papillary microcarcinoma of the thyroid under observation. Thyroid. 2014;24(1):27-34. 